\title{
Emerging Trends in Medical Diagnosis: A Thrust on Nanotechnology
}

\section{Satvekar RK, Tiwale BM and Pawar SH*}

Centre for Interdisciplinary Research, D. Y. Patil University, Kolhapur-416006, India

\begin{abstract}
Nanotechnology is the manipulation of material on an atomic and molecular scale; by changing its physical, chemical and biological properties to produce novel materials, devices, and systems. Nanomaterials exhibit remarkable characteristics such as high surface to volume ratio, catalytic activity, and biocompatibility which make them suitable for various biomedical applications. Nanotechnology have prospective to improve the whole healthcare process for patient; starts from diagnosis to treatment and follow-up monitoring. Medical diagnosis based on nanotechnology provides two major advantages: rapid testing and early diagnosis. The potential contributions of nanotechnology in the medical diagnosis are extremely broad and improve traditional diagnostic tools and methods in the field of clinical diagnosis, imaging and electro- diagnosis. Emerging modalities such as biochip, microarray, nanobarcode, micro-electromechanical systems, lab on chip and nanobiosensor have revolutionized the field of medical diagnosis. Nanoscale materials and nano-enabled techniques are used for diagnosis of various diseases such as cardiovascular diseases, cancer, diabetes, infectious disease, musculoskeletal and neurodegenerative disease etc. Among all medical applications of nanotechnology, nanobiosensor especially enzyme nanobiosensor are sensitive, reliable, robust, reproducible and cost effective diagnostic tool to meet the requirements of healthcare.
\end{abstract}

Keywords: Nanotechnology; Nanomaterials; Medical diagnosis; Imaging; Electrochemical biosensors and Nanobiosensors

\section{Introduction}

Diagnosis of a suspected disease is one of the most critical steps in healthcare and medicine. Diagnosis is the act or process of identifying or determining the nature and cause of a disease or injury through evaluations of patient history, examination and review of a laboratory data [1]. Medical diagnosis is one of the most challenging areas of healthcare and medicine as giving treatment to the patient, careful diagnosis of disease biomarkers is the deciding factor for the treatment of patient. Medical diagnosis should be fast, reliable, specific, accurate, and minimize possibility of 'false positives'. Diagnosis methods with high degree of sensitivity and specificity aid in early detection of diseases and disorders; and hence can provide better prognosis. Technology development for medical diagnosis is one of the important fields of applied science as it straightforwardly affects healthcare of the general population [2].

Many scientists have endeavored to develop more efficient technologies for rapid and accurate diagnosis of diseases. One of the first instances of medical diagnostic device was the stethoscope, which has become the most widely used diagnostic device by clinicians to date. Laennec invented the first medical device: stethoscope for diagnosis which was essentially a simple wooden monaural tube and has been developed over the past two centuries into a binaural flexible device with integrated low pass filtration among other numerous advances. It is responsible for the discovery of new diseases, the creation of criteria for accurate and rapid diagnosis and the development of many new treatments [3]. The subsequent most important tool was the discovery and use of X-Rays by Wilhelm Conrad Rontgen in 1895. Admirably, Rontgen won the first Nobel Prize in physics in 1901 for his discovery which has affected innumerable lives and paved the way for new medical discoveries and diagnostic techniques [4].

Medical diagnosis involves analyzing of symptoms of a disease, determination of tissue type prior to organ transplantation, detection of blood group of patient requiring blood transfusion and to monitor the progression of disease. Numerous techniques and assays are available for diagnosis such as immunoassay, genetic based tests, medical imaging and biosensing. Bioassays commonly used in diagnosis are enzyme linked immunosorbent assay (ELISA), polymerase chain reaction (PCR) based genetic assay and staining assay such as Giesmsa and Gram for viral and bacterial infectious diseases [5]. Conventional diagnostic methods suffer from few limitations occurring as result of low specificity and lack of efficacy. Nanotechnology enhances assays by accomplishing all requirements to provide a platform that is more sensitive than the current gold standard in protein detection, and ELISA [6].

Most biomarker detection is takes place at centralized laboratories using sophisticated automated analyzers, with large waiting time and high costs. Robust, faster and economical devices are extremely desirable for substituting these time-consuming laboratory diagnoses and for diagnostic results available at the patient's bedside i.e. point-ofcare diagnosis. Nanoscale structured materials and devices that can be fabricated today hold great promise for advanced diagnostics and biosensors. Nanotechnology integrates with genomics, proteomics, and molecular machine systems, and attribute to fast, reliable, and onsite effective medical diagnosis. Nanotechnology has revolutionized these modalities by producing novel materials and devices for medical diagnosis as outlined in Figure 1 [7].

\section{Medical Diagnosis}

Medical diagnosis has different modalities like bioassay, biosensors and imaging. Current modalities of diagnosis have limitations such as poor sensitivity, specificity and reproducibility. Diagnostic methods should have greater sensitivity and also facilitate early detection of diseases to provide better prognosis. The conventional diagnostic techniques often cannot identify Alzheimer's, many cancers, and other life-threatening diseases early to offer an effective treatment. But nanotechnology, which is revolutionizing electronics and other fields, promises to similarly renovate medical diagnosis. Nanotechnology

${ }^{*}$ Corresponding author: Pawar SH, Centre for Interdisciplinary Research, D. Y Patil University, Kolhapur-416006, India, Tel: +91 231 2601202, 2601235; Fax: +91 231 2601595; E-mail: pawar_s_h@yahoo.com

Received March 14, 2014; Accepted April 21, 2014; Published April 23, 2014

Citation: Satvekar RK, Tiwale BM, Pawar SH (2014) Emerging Trends in Medical Diagnosis: A Thrust on Nanotechnology. Med chem 4: 407-416. doi:10.4172/21610444.1000172

Copyright: (c) 2014 Satvekar RK, et al. This is an open-access article distributed under the terms of the Creative Commons Attribution License, which permits unrestricted use, distribution, and reproduction in any medium, provided the original author and source are credited. 


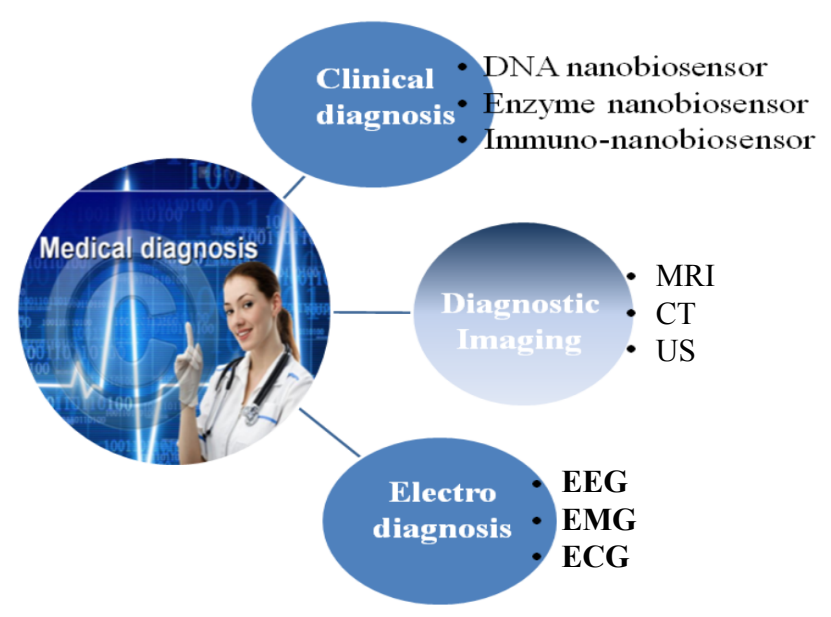

Figure 1: Diverse modalities of medical diagnosis with aid of Nanotechnology.

the engineering of systems at the atomic or molecular scale to construct devices that are elegantly small allows researchers to develop supersensitive nanomaterials that are capable of recognizing disease biomarkers at much lower concentrations than traditional tools [8].

\section{Clinical diagnosis}

The aim of clinical diagnosis should be the rapid testing and complete diagnosis at an earlier stage to provide the potential of curing, possibly with less damage to the patient. It is possible with point-ofcare diagnosis which includes nanobiosensor and nano-scaled devices. Bringing the diagnostic technique with patient i.e. point-of-care; reduces disadvantages of conventional diagnosis and overall cost and time for health care process for patient is reduced extensively. In clinical diagnosis especially in oncology, detection of tumor markers in blood and cell lysates exemplifies the future of cancer diagnostics. The capability to investigate all biomarkers, present at a wide range of concentrations, will support physicians to make earlier diagnosis of malignant diseases which are presently difficult to detect at a treatable stage [9].

Point-of-care diagnosis are seen as integrated devices or tools that can process clinical samples for numerous different types of biomarker in a various settings, such as centralized clinical laboratories, doctor's clinics and at the patient's home. At the hospitals diagnosis is based on either large-scale automated analyzers or bioassays based on ELISA techniques, which are unsuitable for bedside emergency. Presentday home care of diabetic patients should follow self-monitoring in a simple and cost-effective way. Consequently, biosensor technology with new, intelligent, and radically enhanced detection abilities with integrated sample handling to overcome the most common diagnostic problems are greatly required. These devices must minimize sample volumes and lower the limit of detection. Remarkably, such devices could be employed for specific biomarkers viz. thrombin, C-reactive protein, troponin, and so far. Moreover, this could reduce hospital expenses and would be very beneficiary for treatment of patient [10].

\section{Diagnostic imaging}

In vivo imaging is most important in diagnosis of disease which searches for the symptoms of the disease within live tissue suspected of being infected. Medical diagnostic imaging includes MRI, PET, CT, OCT, opto-acoustic or photo-acoustic tomography (OAT/PAT) and NIR imaging [11]. Medical research has exploited the unique properties of nanomaterials for in vivo imaging where tools and devices are being developed by using nanomaterials. Nanotechnology is having impact on this area, particularly by developing molecular imaging agents. A nanoparticle enhances favorable distribution and improved contrast in MRI and Ultrasound images. Nanomaterials containing contrast agents can greatly improve sensitivity of diagnostic imaging techniques. Nanomaterials can improve imaging techniques even at single cell before any symptoms appear [12].

Super-paramagnetic nanoparticles are widely used as MRI contrast agent, while liquid perflurocarbon nanoparticle and liposome are examples of ultrasound contrast agent. Optically absorbing gold nanoparticles can be used as contrast agent for opto-acoustic imaging as well as gold nanoparticles used in NIR imaging due to their lumiscence properties. Body cannot clear them due to their nanosize, which prolongs the time span for imaging. Traditional fluorophores such as organic dyes and fluorescent proteins suffer from several intrinsic problems including rapid photo-bleaching, spectral cross-talking, narrow excitation profiles, and limited brightness/signal intensity. These shortcomings can be reverted by iron oxide nanoparticles (MNP) coated with peptides specifically bind to the tumor cell which in turn enhances the images of MRI owing to its magnetic properties [13]. MNP-MRI allowed noninvasive, real-time quantification of pancreatic inflammation for diagnosis of autoimmune diabetes [14].

\section{Electro diagnosis}

Electrodiagnosis is field of diagnosis which encompasses electrophysiology and electrical technology to study human neurophysiology, neurodiagnosis, electromyography and evoked potential. It is method of obtaining information about disease by passively recording the electrical activity of body parts or by measuring their response to external electrical stimulus. The most widely used specialized diagnostic technique of recording spontaneous electrical activity are electrocardiography (ECG) to monitor electrical activity of heart and elctrocephalography (EEG) to assess electrical activity of brain, electromyography (EMG) for monitoring nerve and muscle cell activity [15], tonometry for measuring internal eye pressure and spirometry to measure lung function. ECG electrodes with integrated nanostructure conformal antenna used as wireless transmitter to send data for remote health monitoring.

\section{Nanotechnology for Medical Diagnosis}

\section{Nanotechnology}

The prefix "nano" derived from Greek word "dwarf", while the term "nanotechnology" was coined by the Japanese researcher Norio Tangiuch in 1974 [16]. However, the concept of nanotechnology was realized by the famous physicist Richard Feyman in 1959 in his landmark lecture in which he mentioned the possibility of manipulating materials at the level of individual atom and molecules [17].

Nanotechnology is promising field of research because of its potential to revolutionize each field as it deals with the properties of materials at the nanoscale dimension between 1-100 nm. Nanotechnology provides new materials with novel properties and function for various biomedical applications such as diagnostics, drug delivery, therapy, tissue engineering, hyperthermia treatment, and biosensors [18]. As a substantial branch of science, it has the most vital role in field of human healthcare and medicine. Nanotechnology in medical diagnosis covers all field of science for imaging, measuring, and manipulating matter at the nanoscale and has important application in diagnosis, prevention and treatment [19].

Biomedical application of nanotechnology has ability to enable early detection, prevention, treatment and follow up of many life- 
threatening disease including cancer, cardiovascular disease, diabetes, Alzheimer's and AIDS as well as infectious disease [20]. The most auspicious application of nanotechnology is in Alzheimer's disease because patients are diagnosed after they pass away and their brain is examined for the telltale damage, scientists are investigating for tests that would help make a diagnosis in living patients [21]. Nanotechnology enables the development of novel therapies and improves traditional treatment and diagnosis process by manipulating atom and molecules to produce nanostructures of same size as biomolecules for interaction with human cells. Interpreting, reproducing and modulating medical diagnosis in assistance with nanotechnology in a bid to make lives healthier is one of the aims of the modern nonscientists by diagnosis of disease: nanotechnology will provide better diagnostic tools to look for biomarkers that recognize possible complications before they occur.

\section{Nanomaterials for medical diagnosis}

Nanomaterials exhibit higher chemical reactivity, increased mechanical strength, faster electrical and magnetic responses owing to its high surface to unit volume ratio. The properties of nanomaterials are very different than their bulk materials, so amenable to diagnostic technique. Nanoparticles can attach to biomolecules, allowing detection of disease biomarkers in a lab sample at a very early stage. Nevertheless, nanomaterials possesses electrical conductivity, catalytic properties, good stability and high loading of biomolecules owing to it high surface to volume ratio. Because of their small size, nanomaterials can readily interact with biomolecules and gaining access to so many areas of the human body. Nanomaterials offer the opportunity to interact with cells at the molecular scales in real time, and during the earliest stages of a disease [22].

There is variety of methods to synthesize NPs such as physical, chemical and biological synthesis. The common ways to produce nanomaterials are top down and bottom up techniques. The topdown technique is start with a bulk material and then breaks it into smaller pieces using mechanical, chemical or other form of energy. An additional way is to synthesize the material from atomic or molecular species via chemical reactions, allowing for the precursor particles to grow in size, which is called bottom-up technique [23]. Biological synthesis is green; eco-friendly synthesis of NPs from microorganisms has been greatly facilitated for biomedical applications [24].

Nanomaterials become a platform for fabrication of novel diagnostic tool and revolutionized diagnostic process. The diverse and enhanced properties of nanomaterials have been exploiting for medical diagnosis e.g. increased relative surface area, the emergence of quantum effects and interactions with biological systems which presents opportunities for scientists. Noteworthy, assets that mark nanomaterials as exciting material, such as increased reactivity and the potential to cross cell membranes have positive impacts on healthcare. Overall process of application of nanotechnology from nanomaterials to nano-enabled tools in medical diagnosis is summarized in Figure 2.

Magnetic Nanoparticles (MNP): MNPs are employed in multiple disciplines such as biosensors, magnetic resonance imaging and nanoelectronics etc. MNPs commonly consist of magnetic element such as iron, nickel, and their derivatives. MNPs are versatile diagnostic tool as it is manipulated using external magnetic field. This 'action at a distance' phenomenon combined with intrinsic penetrability of magnetic field into human tissue enables their detection in vivo using MRI [25].

Precise detection of protein biomarkers and pathogens in clinical samples is vital early diagnosis of diseases, monitoring of treatment, and design of personalized medicine. In general, biomolecules have insignificant magnetic susceptibility. Hence, MNP for biosensor enhance sensitivity and effectively reduce sample preparation requirements. Magnetic sensors, such as magnetic relaxation switch assay sensors, magneto-resistive sensors, and magnetic particle relaxation sensors have been developed [26].

Super-paramagnetic iron oxide nanoparticles (SPION) are made of an iron oxide core and coated by either inorganic materials like silica or organic materials such as phospholipids, natural polymers such as dextran or chitosan. SPIONs are a versatile agent for early diagnosis of cancer, atherosclerosis and other diseases. Moreover, SPIONs are used as contrast agents for MRI imaging and as an in-vitro application in bioassay by means of a vehicle for the detection of biomarkers [27]. When SPION used in biosensors it improves the sensitivity and selectivity of diagnosis.

Quantum Dots (QD): QDs are spherical, fluorescent nanocrystals made up of semiconductor materials of intermediate size typically between 2-8 $\mathrm{nm}$ in diameter; are indebted to unique properties such as, narrow sized tunable emission spectra, broad excitation spectra, negligible photo bleaching, high sensitivity and stable fluorescence with simple excitation without the need of laser. QDs are widely used as alternative to conventional flurophores and for development of biosensors to detect biomolecules such as proteins, neurotransmitters, enzymes and amino acids [28]. Bioconjugated QDs have the potential to be used in cancer diagnosis due to its bright and stable fluorescent light emission and sensitivity of fluorescence imaging.

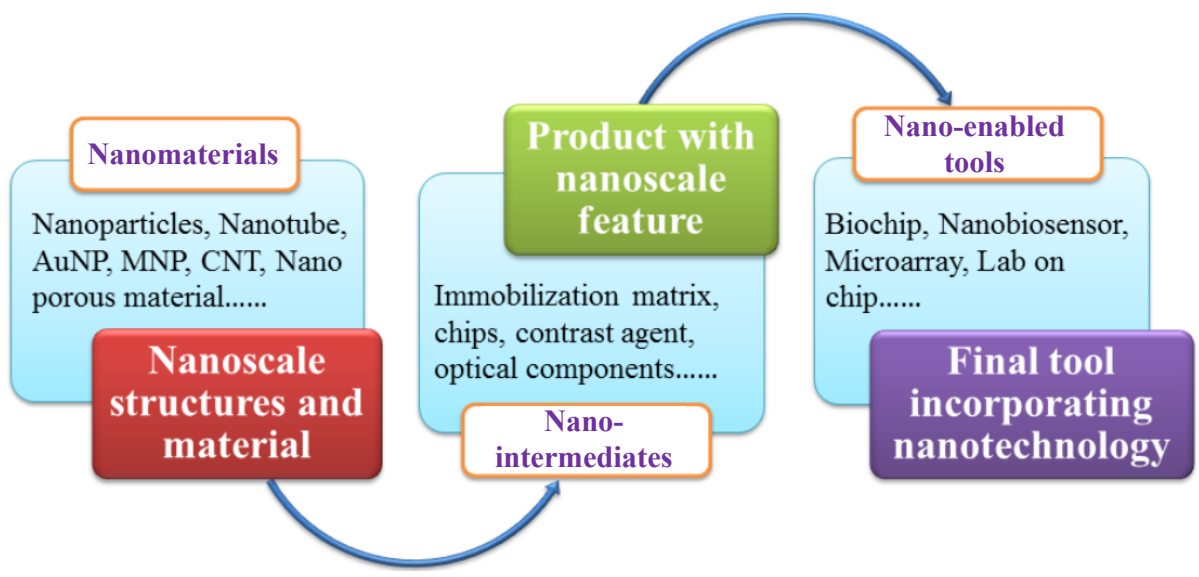

Figure 2: Schematic representation for process of nanoscale materials in medical diagnosis. 
Moreover QDs are ideal candidate for biosensor application supports continuous monitoring of signals [29]. QDs may be used in the future for locating cancer tumors in patients and in the near term for performing diagnostic tests in biological samples [30].

Carbon Nanotube (CNT): CNT have been most extensively used nanomaterials in biosensors and diagnosis. CNT are long, hollow cylindrical carbon structures composed of one, two or several concentric graphite layers capped with fullerenes hemispheres which are referred as single, double and multi-walled CNTs. They have unique mechanical, optical, and electronic properties with high electrical and thermal conductivity. Furthermore CNT have high aspect ratio, unique optical property and small sizes exhibit potentials for a variety of biomedical applications, including the diagnosis of cancer, and infectious diseases. These prospective applications are encouraged by their capability to penetrate biological membranes and relatively low toxicity [31]. For diagnostics, CNT can help to detect a protein biomarker of disease when fabricated on the surface of a nanobiosensor. When it binds with a protein, the nanotubes change their electrical resistance which can then be measured to determine the presence of a particular protein, for example serum protein biomarkers that can indicate breast cancer [32]. CNTs have potential to increase the speed of biological sensors by reducing the biosensor response time [33].

Graphene oxide (GO): GO is thin layer of $\mathrm{sp}^{2}$ hybridized carbon, extensively used for medical diagnosis due to its exciting properties viz. tunable band gap, high elasticity, high mechanical strength, very high room temperature quantum Hall effect, high electron mobility and high thermal conductivity [34]. GO is a transparent material with low production cost and minimum environmental impact; so extensively employed in electrochemical biosensors. The sheets of GO, on which attached antibody binds to the cancer cells which then tag the cancer cells with fluorescent molecules to make the cancer cells stand out in a microscope. Besides, it can detect a very low level of cancer cells, as low as 3 to 5 cancer cells in a one milliliter of blood sample [35].

Gold Nanoparticles (AuNPs) and Silver Nanoparticles (AgNPs): Gold and Silver metals are real jewels beyond that the nanosized AuNPs and AgNPs are more precious and in great demand by scientists. AuNPs are most attractive and extensively studied nanomaterials in bio-analytical field for medical diagnosis, owing to its fascinating features such as ease of synthesis, high biocompatibility and non-cytotoxicity. AuNPs have biomedical applications in the areas: labeling and biosensing. For labeling, certain properties of the particles are exploited to generate contrast. Their nano size and the possibility of functionalization with biomolecules mean that they also provide extremely high spatial resolution and specificity in many labeling applications. Moreover, the properties viz. optical properties, strong absorption, scattering and especially Plasmon resonance make them of value for a large variety of light-based techniques including combined schemes such as photo-thermal or photo-acoustic imaging. Finally, gold nanoparticles can also be used in biosensors as their optical properties can change upon binding to certain molecules, permitting the recognition and quantification of analyte. The sensitive DNA detection can be exploited by property to change absorption spectra drastically of gold nanoparticles when several particles come close to each other [36]. The Silver nanorods in a diagnostic system are being used to separate viruses, bacteria and other microscopic components of blood samples, allowing clearer Raman spectroscopy signals of the components. This method has been demonstrated to allow identification of viruses and bacteria in less than an hour [37].

Porous nanomaterial: The porous nanomaterials are particularly promising for fabrication of optical biosensors as it possesses wide range of physical characteristics such as high purity, tunable porosity, nanoscale structuring, high photochemical, physical rigidity and thermal stability. Moreover, optical transparency is the most significant features offered by porous nanomaterials for creating highly sensitive and selective matrices to integrate in biomedical applications. Porous nanomaterials retain native conformation and reactivity of biomolecules. The greater optical transparency makes it valuable in the application of biosensor for medical diagnosis [38].

There are several efforts has been taken by scientist to develop nanomaterials based disease detection systems which are summarized in Table 1.

\section{Emerging trends in medical diagnosis}

Biochip: Biochips promised immense help in the field of medical diagnosis as they are miniaturized laboratories that can perform hundreds of simultaneous biochemical reactions. Biochip technology has a promising perspective in the rapid diagnosis of clinical disease with the features of high sensitivity and specificity. DNA biochips are produced by in situ synthesis of oligonucleotides or peptide nucleic acid (PNA) or spotting of DNA fragments on chip which allows the monitoring of expression of mRNAs or the occurrence of polymorphisms in genomic DNA due to the hybridization of RNA or DNA derived samples. Basic types of DNA biochips are the expression chip, sequencing chip, and chips for comparative genomic hybridization [47]. The application of gold nanoparticles

\begin{tabular}{|c|c|c|c|c|}
\hline Sr.No. & Nanomaterial & Nanoplatform and Significance & $\begin{array}{l}\text { Sensitivity, Linear range (LR), and limit of } \\
\text { detection (LOD) }\end{array}$ & References \\
\hline \multirow{2}{*}{1} & \multirow{2}{*}{ Magnetic nanoparticles } & $\begin{array}{l}\text { Magnetic multiplexed biosensor nanoplatform: } \\
\text { Magnetic properties, robust, ultrasensitive }\end{array}$ & LR:10 100,000 pg/mL, LOD: CRP 0.6 pg/mL; & [39] \\
\hline & & $\begin{array}{l}\text { Magneto-DNA assay: } \\
\text { improved accuracy, rapid and specific profiling of pathogens }\end{array}$ & $\begin{array}{l}\text { Sensitivity } 1 \text { bacterium } \\
\text { LOD: } \sim 0.5 \text { pM [DNA] }\end{array}$ & [40] \\
\hline 2 & Quantum Dots & $\begin{array}{l}\text { Potentiometric biosensor } \\
\text { zero-dimensional electronic properties }\end{array}$ & $\begin{array}{l}\text { Sensitivity: } 97 \mathrm{mV} / \text { decade, LR: } 1 \times 10^{-6} \mathrm{M}-1 \times \\
10^{-3} \mathrm{M}\end{array}$ & [41] \\
\hline 3 & Carbon Nanotube & $\begin{array}{l}\text { Enhanced enzyme loading, higher aspect ratio, and excellent electro- } \\
\text { catalytic activities }\end{array}$ & $\begin{array}{l}\text { Sensitivity: } 6 \mu \mathrm{AmM}^{-1} \mathrm{~cm}^{-2} \\
\text { LR:0.02 to } 6 \mathrm{mM} \text {, } \\
\text { LOD: } 0.01 \mathrm{mM}\end{array}$ & [42] \\
\hline 4 & Graphene oxide & large surface-to-volume ratio and good electrochemical activity & $\begin{array}{l}\text { LR: } 10 \mathrm{fM} \text { to } 50 \mathrm{nM} \text {, } \\
\text { LOD: } 10 \mathrm{fM}\end{array}$ & [43] \\
\hline \multirow[t]{2}{*}{5} & \multirow{2}{*}{$\begin{array}{l}\text { Gold and Silver } \\
\text { nanoparticles }\end{array}$} & $\begin{array}{l}\text { Electrochemical enzyme biosensor } \\
\text { good catalytic properties }\end{array}$ & $\begin{array}{l}\text { LR:0.0100 - } 5.00 \mathrm{mmol} \mathrm{L}^{-1} \\
\text { LOD: } 4.3 \mu \mathrm{mol} \mathrm{L}^{-1}\end{array}$ & [44] \\
\hline & & surface conductivity & LR: 0.72 to 25.92 and 1.62 to $19.62 \mu \mathrm{M}$ & [45] \\
\hline 6 & Porous nanomaterial & $\begin{array}{l}\text { FRET-based biosensor } \\
\text { Fluorescence property }\end{array}$ & LOD: $10-5 \mathrm{ng} \mathrm{mL}^{-1}$ & {$[46]$} \\
\hline
\end{tabular}

Table 1: Nano-platforms and their significance in Medical Diagnosis. 
as oligonucleotide labels instead of fluorescence dyes and enzymeconjugation in DNA detection assays; provided new insight into the application of nanoparticles in biochips. The gold- labeled probes were compatible with gene-chip and can be used as an alternative marker because of their high stability, high labeling density, superior sensitivity and lower expense [48]. Biochips enable researchers to quickly screen large numbers of biomolecules for disease diagnosis. The biochip uses plasmonic interferometers and used to measure blood sugar levels by measuring glucose concentrations in saliva instead of blood. This technique takes advantage of a convergence of nanotechnology and surface plasmonic, which explores the interaction of electrons and photons to detect very low concentrations of glucose in saliva [49].

Microarray: Microarrays are promising for competent and personalized approaches to human health care. Microarray offer a powerful tool for screening thousands of proteins at a time, where variety of biomolecules such as antibodies and enzymes are immobilized in an array format on glass slide [50]. The surface of the glass slide is then probed with sample of interest that binds to relevant antibodies on chip which will be analyzed by detection method on microarray. Profiling proteins on arrays will be used in distinguishing the proteins of normal cells from early stage cancer cells and malignant metastatic cancer cells. Likewise, it also helps to sensing of the vast amount of genomic information and its application for early detection of genetic diseases. The applications of antibody microarray to cancer diagnosis are growing in scope and efficiency. Enhancement in microarrays miniaturization with the aid of nanotechnology will further contribute to molecular diagnostics and the development of personalized medicine [51].

Nanobarcode: Nanobarcode act as encoded substrates in multiplexed assays which are metallic, durable, encode-able and machine-readable submicron-sized tags [52]. These are freestanding, cylindrical nanoparticles with specific patterns of submicron stripes of noble metal ions; produced by alternating electrochemical reduction of the appropriate metals. The differential reflectivity of the adjacent metals provides contrast black and white stripes which makes them unique like conventional barcodes under light, or fluorescent microscopy, or mass spectrometry [53]. Nanobarcode is advantageous in coding in multiplexed assays for proteomics, Single-nucleotide polymorphism (SNP) mapping, and in point-of-care handheld devices.

The nanobarcode assays are a nanotechnology-based technique for detecting proteins which shows extraordinary sensitivity in detecting certain antigens at extremely low concentrations, now devoid of the use of the polymerase chain reaction (PCR). This assay uses disease biomarkers that cannot be used in the conventional assays. ADDLs are considered neurotoxins in Alzheimer's disease (AD) pathogenesis clinically detected by a new diagnostic approach for $\mathrm{AD}$ using the nanobarcode assay [54].

Lab-on-chip: Lab-on-a-chip devices are 'miniaturized integrated laboratories' that allow separation and analysis of biological samples. These are composed of micro-fluidic systems including micro-pumps and micro-valves integrated with microelectronic components [55]. Nanotechnology allows miniaturization of these devices which requires high electrical fields strengths that can be obtained by using nanosized electrodes and the membranes based on nanopore separation systems. Lab-on-chip devices have rapidly evolved for applications in a number of clinical analysis operations which is fabricated on silicon or glass substrates using thin film technology and photolithography techniques. The advantages of Lab-on-chip devices are the small sample and reagent volume employed in these systems, rapid response time, less sample wastage, and prospect of developing disposable devices.
Micro-electro-mechanical systems (MEMS): The difference between MEMS and micro-fluidic systems are that MEMS do not require reagents or a fluidity based substrate to react upon. Even though MEMS are primarily used in drug-delivery systems, one primary application of MEMS in diagnostics are the swallowed capsule technology pills that allow doctors to visualize GI bleeding. The patient swallows a capsule containing a light-emitting diode for illumination, a CMOS (complementary metal-oxide semiconductor) video camera and optics for taking images, a battery, and a transmitter". Implantable devices will be able to continuously sense and adjust the body's chemical balance, in the bloodstream or in specific tissues [56]. Furthermore, implanted sensors will be valuable in acquiring a continuous record of the person's state of health and allow more sensitive adjustment of the body's state, and earlier detection of diseases. The MEMS allow separations, chemical reactions, and calibration-free analytical measurements to be performed directly in very small quantities of complex samples such as whole blood.

Nanobiosensor: Biosensors are widely used in medical diagnosis to scrutinize or detect biomarker for diagnosis of various diseases starting from diabetes to cancer. The recent progress in biosensor fabrication allows diagnosis of life-threatening diseases more reliably. Furthermore, miniaturization of biosensor meets the requirements of potable and cheap diagnostic tool for resource poor settings for disease such as HIV/AIDS. Nanotechnology provides great opportunities to improve the sensitivity, stability and anti-interference ability of the biosensor systems. With the progress in nanotechnology, various novel nanomaterials have been invented and their novel properties are being gradually reveals which greatly enhances biosensor performance. The small sized nanoparticles overcome the limitation of miniaturization, leading to lower detection limits, even getting zepto-molar concentrations. Noteworthy, nanoparticles can produce a synergic effect between conductivity, catalytic activity, and biocompatibility to enhance the signal transduction. Most prominently, nanoscale materials are used in biological sensors in the detection of biomolecules. Nanomaterial based biosensing involve in vivo diagnosis with high sensitivity, less cytotoxicity, and long-term stability for early screening of biomarkers and reliable point-of-care diagnosis [57]. Nanobiosensors have made a great deal of excitement due to their capacity to sense a wide range of biomolecules at incredibly small concentrations.

\section{Nanobiosensor for Clinical Diagnosis}

\section{Biosensor}

Among all biomedical applications of nanotechnology, biosensor represents one of the most emerging field of research for which nanomaterials provides enhanced sensitivity, stability and miniaturization i.e. biosensor research received enormous benefits from nanotechnology. Biosensors based on nanomaterials such as nanoparticles, nanotubes, nanowire and nanocomposite have various advantages such as sensitivity, stability, reproducibility as well as continuous and insitu monitoring in matrix. A successful biosensor should be small, cheap, reliable and portable, to reach the interest of millions of patient for diagnosis of diseases and disorders. In this context, minimize blood volume and avoid painful sampling are most important challenges for biosensor fabrication [58]. Mostly disposable biosensor ensures bypass of contamination and simpler fabrication while implantable biosensors provide more accurate in-situ monitoring of disease.

IUPAC proposed definition of Biosensor as, "A biosensor is selfsufficient integrated device, which is capable of providing specific 
quantitative or semi quantitative analytical information using a biological recognition element (biomolecules viz. Enzyme, DNA, and Antibody), which is retained in direct spatial contact with a transducer element" The biomolecules such as enzymes, antibodies, nucleic acids, cell and bio-mimic component employed as biorecognition element which is highly favorable owing to its specificity and catalytic activity. These are in intimate contact with a physiochemical transducers in biosensor system. The transducer element may be electrochemical, piezoelectric, optical, and thermometric which enable to convert a biochemical signal into an electric signal [59].

Principle of biosensor: Biosensor comprises of a biorecognition element in intimate contact with a proper transducer. The biorecognition element gives rise to signal as the biochemical reaction of interested analyte, which is detected by transducer to give electrical signal. This reaction between the biomolecule and the substrates produces a product in the form of electrochemical, heat, light or sound, and then a transducer viz. electrochemical, semiconductor or thermistor changes the product of the reaction into readable data.

\section{Biosensor Components}

\section{Biosensor consists of three components:}

i) The biorecognition element e.g. Enzymes, Antibodies, Nucleic acids, cell lysates, Microorganisms, Cell receptors, etc.

ii) The transducer which acts as an interface, measuring the physical change that occurs with the reaction at the biorecognition element and then converting that energy into measurable electrical output. These transducers are optical, electrochemical, opto-electronic, piezoelectric, Thermal and Mass.

iii) The detector element which passed signals from the transducer to a microprocessor where they are amplified and analyzed.

Generation of biosensor: One of the earliest research on the concept of appeared in 1962 when first glucose biosensor developed by Clark and Lyons based on amperometric glucose biosensor to detect oxygen pressure [60]. Since then many biosensors have been commercially exploited which offers a more sensitive, specific, rapid, real and reproducible measurements. In recent times nanobiosensor, implanted biosensors and integrated biosensors are in current research and development.

Biosensors can also be classified based on their innovation order into first generation which is the simplest approach involving direct detection of either increase of an enzymatically generated product or decrease of a substrate of a redox enzymes using natural mediator for electron transfer e.g. glucose biosensor which uses enzyme glucose oxidase and oxygen detecting decrease in oxygen level or increase in hydrogen peroxide corresponding to the level of glucose. First generation biosensor relies on the use of natural oxygen as cosubstrate, and detection of hydrogen peroxide produced in reaction as shown in Figure 3. In second generation biosensor, oxygen gets replaced with non-physiological electron acceptor or mediator which is able to shuttle electrons from redox center of enzyme to the surface of electrode. Such meditation is dependent on concentration of analyte in real sample. The artificial redoxmediators like ferrocene, ferricynide and quinones for electron transfer increases the reproducibility and sensitivity. Finally, third generation in which the redox enzymes are immobilized on the electrode surface in such a manner that direct electron transfer is possible between the enzyme and surface of electrode.

The prospects for biosensors based on a specific requirement in clinical diagnostics are considered to be in the following areas [61]:
* Direct electron transfer in redox proteins and enzymes
* Optimization of molecular interactions
* Micrometer and nanometer scale performance of diagnostic devices
* Device design for whole blood biosensors
- Biocompatibility studies for in vivo biosensors
* Packaging for in vivo biosensors
* Micro-fabrication techniques for mass production of biosensors
* Immobilization and protection of biomolecules on multi- analytic devices
* Signal interference in biological samples
* Bed-site monitoring
* In home test kits

\section{Types of nanobiosensor based on transducer mode}

Biosensors can be generally classified into two criteria's based on sensing element and transduction modes. Transduction mode depends on the physiochemical change resulting from sensing element. Hence on the basis of different transducers, biosensors can be electrochemical (amperometric, conductometric and potentiometric), optical (absorbance, fluorescence and chemiluminense), piezoelectric (acoustic and ultrasonic) and calorimetric [62]. The major types based on transducer mode are discussed below:

\section{Electrochemical biosensor}

Amperometric nanobiosensor: Amperometric biosensor is based on the electrochemical analysis in which the signal of concern is a current that is linearly dependent upon the concentration of the biomarker. The signal transduction process is accomplished at a fixed potential between the working electrode and a reference electrode, and measuring the current as a function of time which is a direct measure of
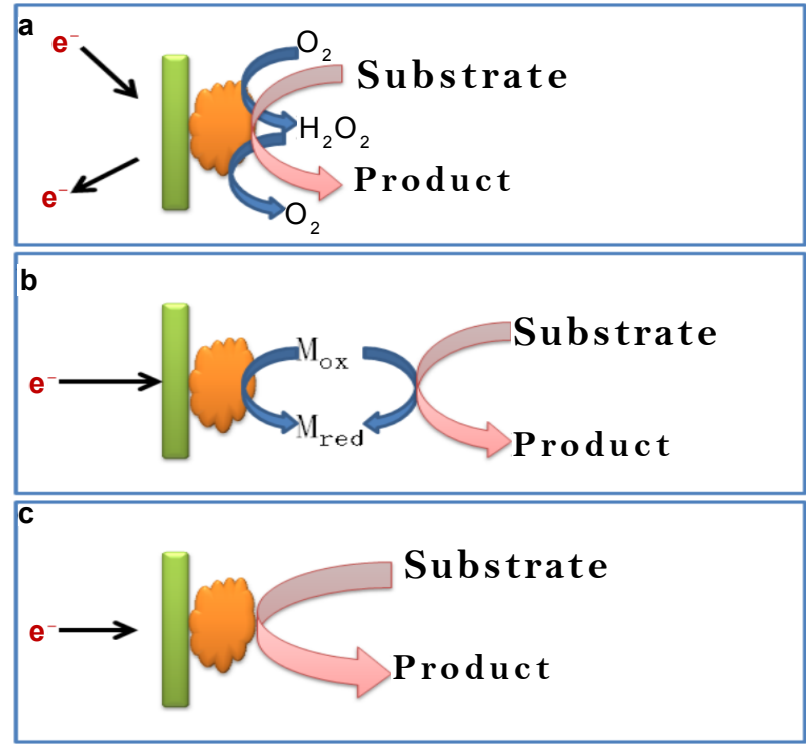

Figure 3: Schematic representation of the three generation of biosensors (a) first generation (b) second generation and (c) third generation. 
the rate of electron transfer. In most cases the biorecognition element is immobilized on the working electrode. The analyte is oxidized or reduced at the working electrode and the current flow directly proportional to the concentration of the electro-active species [63].

Potentiometric nanobiosensor: In potentiometric sensors, the potential difference between the reference electrode and the indicator electrode is measured without polarizing the electrochemical cell. The analytical information is achieved by transforming the biorecognition process into a potential signal. A permselective ion-conductive membrane and high impedance voltmeter is normally utilized to measure the potential signal, which arises when the analyte interacts with the surface. The electrical potential difference or electromotive force (EMF) measured between two electrodes at near zero current. The indicator electrode or an ion-selective electrode (ISE) develops a change in potential as a function of analyte concentration in sample [64].

Conductometric nanobiosensors: These are based on the measurement of electrolyte conductivity, which varies when the cell is exposed to different environments. Conductivity measurements are generally accomplished with AC supply which is a linear function of the ion concentration. Conductometric-based biosensors couple conductance and a biorecognition event in which mostly reactions involve a change in the ionic species concentration and this can lead to a change in the solution electrical conductivity. The major problem with this technique is that the sensitivity is generally lower compared to other electrochemical methods [65].

Optical nanobiosensor: Optical biosensors (optodes), have received considerable attention for diagnosis. The optical biosensors involve direct detection of the analyte of interest or indirect detection through optically labeled probes. In general, there are at least four types of biosensors using the principles of optical technology. These are as follows: absorption, reflection, chemiluminescence, fluorescence, and phosphorescence. Optical biosensors are potent alternative to conventional analytical techniques because these are highly sensitive, reproducible, rapid, and simple-to-operate. The optical biosensor design encompass direct detection of the interested analyte or indirect detection through optically labeled probes, and the optical transducer may detect changes in the absorbance, luminescence, polarization, and refractive index [66].

Piezoelectric nanobiosensors: These biosensors are mass sensitive detectors, which has the principle that an oscillating crystal resonates at the natural resonance frequency. The piezoelectric materials are able to generate and transmit acoustic waves. When a piezoelectric biosensor surface is coated with a biomolecule (antibody) and placed in a solution containing the pathogen, the attachment of it to the antibody coated surface results in an increase in the crystal mass, and this shows a corresponding frequency shift. This mechanism is relatively simple, inexpensive, and offers direct label-free analysis [67].

Calorimetric nanobiosensor: Most chemical and biochemical processes involve the generation of exothermic heat which is used as a basis for measurement of rate of reaction and ultimately analyte concentration. The fabrication of the Calorimetric biosensor normally consist a thermistor or thermopile employed as a temperature transducer to determine heat changes [60]. The device is covered with the enzyme and when this interacts with the analyte it generates an exothermic reaction, which is recognized as a heat change. Obvious benefits of this biosensor are that it can be easily miniaturized, can be used in turbid samples, and it is a label-free approach [68].

\section{Types of nanobiosensor based on biorecognition element}

Sensing elements include enzymes, antibodies (Immunosensor), micro-organisms (whole cell biosensors), and DNA.The main types based on are as follows:

DNA Nanobiosensor: The DNA nanobiosensor is based on the conversion of the base-pair recognition event or hybridization event (the complementarities of adenine-thymine and cytosine-guonosine pairing in DNA) into a measurable electrical signal. DNA duplex formation or hybridization event forms the basis of electrochemical detection in which electrochemical signals are generated and enhanced by covalent binding of nanomaterials to the DNA probe. DNA probe is the known sequence of bases which can be synthesized and labeled with optically detectable compounds. DNA biosensors are utilized as suitable candidate for rapid and reliable diagnosis of genetic diseases, the detection of pathogenic biological species [69].

Immuno-nanobiosensor: Biosensors which monitor antigenantibody interactions are referred to as immunosensor. Immunosensor monitor antigen-antibody interaction in which either antigen or antibody is immobilized on a solid-state surface of electrode and participate in biospecific interaction with the other component, allowing detection of desired biomolecule. Antigen-antibody reactions are very specific and a very minute concentration of target molecule in the body can be detected and easily screened [70]. Immunoreactions are known for their high sensitivity and selectivity so most reliable tool for clinical diagnosis. Optical and electrochemical detection strategies are mostly used in immunosensor.

Enzyme nanobiosensor: Enzyme nanobiosensor plays a vital role in diagnosis of various diseases which are composed of enzyme as biorecognition component that uses their catalytic activity for detection of analyte. The success of any enzyme sensor depends on enzyme loading, the use suitable $\mathrm{pH}$ and type of immobilization method and moreover, how well it retains its enzymatic activity on transducer. The most important part of a biosensor fabrication is the immobilization of a desired enzyme [71]. However, the usefulness of immobilized enzyme on electrodes depends on factors such as the immobilization method, the chemical and physical conditions $(\mathrm{pH}$, temperature and contaminants), thickness and stability of the membrane used to couple the enzyme.

Whole cell nanobiosensors: Whole cell biosensors are utilizes living cells or Microorganisms such as bacteria and fungi as the biorecognition element which can detect the intracellular and extracellular conditions, physiological biomolecules to produce a response. The detection limit of these biosensors is mainly measured by the environmental conditions in which the cells can remain alive for long period. However the major drawback of whole cell biosensor is the stability of the whole cell, which depends on several conditions such as the lifetime, sterilization, and biocompatibility etc. Despite these consequences the whole cell biosensor is still promising among the researcher due to the advantages over the other biosensors [72].

\section{Enzyme Nanobiosensor}

Enzymes are used reliably in the medical field, such as in the diagnosis and treatment of various diseases. For example, enzymes are used in biosensors for the detection of various biomarkers such as glucose, cholesterol, uric acid. Enzymes are most favorable candidate as a biorecognition element in the fabrication of biosensor because of its selectivity and sensitivity compared to conventional catalyst. However, enzymes are prone to the microenvironment $(\mathrm{pH}$, temperature) and have a short operational lifetime. Moreover, in aqueous solution, 
enzymes lose their catalytic activity rapidly due to the oxidation reaction or their tertiary structure can be destroyed in air-water interface, these shortcomings can be avoided by immobilization of enzymes. Immobilized enzymes are more robust and advantageous such as convenient handling, more resistant to environmental changes and increase the affinity for the substrate [73].

The detection limit of enzymatic biosensors is generally determined by the enzyme's activity. However, the major drawback with enzymatic biosensors is the stability of the enzyme, which be influenced by conditions such as temperature, $\mathrm{pH}$ and buffer etc. The capability to conserve enzyme activity for a long period of time up till now remains a major obstacle. Regardless of these pitfalls, the enzymatic biosensor is still the most usually used biosensor, and this is essentially due to the prerequisite for monitoring glucose, cholesterol, uric acid, lactate for diagnosis of various diseases in blood.

\section{Enzyme Nanobiosensors for clinical diagnosis}

Glucose: The abnormal level of glucose related to diabetes and the risk for renal, retinal, and neural complications. Thus the measurement of glucose in human blood is important for diagnosis of diabetes and other diseases. Glucose biosensors based on glucose oxidase have been dynamically investigated as the potential biosensor with wide range of analytical techniques [74].

Cholesterol: The increasing prevalence of cardiac arrest and cardiovascular diseases are major reason of death in human worldwide. The cholesterol level detection in human blood is of great significance in clinical diagnosis as high cholesterol in blood is associated with coronary heart disease, hypertension, myocardial infarction, brain thrombosis, arteriosclerosis, lipid metabolism dysfunction, etc. [75]. In conventional assays, cholesterol determination is performed by enzymes, such as cholesterol oxidase and cholesterol esterase which can be used to monitor both free and esterified cholesterol levels.

Uric acid: A major nitrogenous compound of urine is uric acid which is the product of purine metabolism in the human body. It is correlated to many clinical disorders; high levels of uric acid in the blood are associated with gout, hyperuricemia, Lesch-Nyhan syndrome and other conditions including increased alcohol consumption, obesity, high cholesterol, diabetes, high blood pressure, kidney disease and heart disease.

Lactate: The level of lactic acid in blood is used in clinical diagnosis of hypoxia, lactic acidosis, some acute heart diseases. Trustworthy, blood lactate detection method would also be of interest in sports medicine.

Urea: Urea is generally monitored in blood sample to obtain evidence of kidney disease. It is the best biomarker for evaluating the level of uremic toxins in body. Eggshell membrane is a natural materials used as a membrane for immobilization of urease enzyme for the development of a potentiometric urea biosensor and showed good operational and storage stability [76].

\section{Fabrication of enzyme nanobiosensor}

The basic requirements for an enzymatic nanobiosensor are: (1) an enzyme which acts on its substrate to produce a molecule which is capable of being reduced or oxidized at an electrode surface; (ii) a immobilization technique for the enzyme to ensure close proximity of enzyme to the electrode which retains the functionality of the enzyme; (iii) an electronic system proficient in controlling the potential of the electrode and measuring the current produced in the redox reaction. For the immobilization of enzyme chemical and physical methods such as covalent binding, absorption, cross-linking, entrapment, and encapsulation have been used. These techniques suffer from some disadvantages like leaching of biomolecules and loss of activity. Accordingly, choice of the immobilization materials and methods should be carefully considered to maintain the enzyme activity for a long time under various conditions. The fabrication of biosensor is dependent on factors like prerequisite of analysis, techniques employed and cell configuration. Conventionally, bulky electrodes and "beaker-type" cells configuration have been utilized. But nowadays, micro-fabrication permits the replacement of these traditional cells configuration and bulky electrodes with appropriate sensing devices. The innovative techniques such as thick and thin film technology, Screen-printing technology and photolithography are used in fabrication of biosensors for clinical diagnosis [77].

The fabrication technique which is well suited for mass production and portable devices allow both in situ and real time monitoring. Perhaps, disposable screen-printed strips are commonly used by diabetic patients for self-monitoring of blood glucose levels. Such fabrication method offers mass production of really inexpensive and hitherto highly reproducible enzyme biosensor.

\section{Conclusion}

Nanotechnology is influencing more deeply on medical research; new and safer diagnostic tools are available which are enabling us to diagnose a disease very earlier as it onsets, and to develop diagnostic strategy against it. Forthcoming years, nanotechnology will continue to evolve and expand in many areas of human life and its achievements will be applied in medical sciences for diagnosis and patient treatment. Nanotechnology has revolutionary opportunities to fight against many diseases such as diabetes mellitus, cancer, neurodegenerative diseases; as well as identifying the microorganisms and viruses associated with infections. Existing and conventional technologies for medical diagnosis may be reaching their confines. Nevertheless, nanotechnology would provide assistance to make diagnosis of diseases more sensitive, rapid and create diagnosis tools easier to use, allowing doctors to identify disease earlier and begin treatment earlier.

Nanotechnology for medical diagnosis is most innovative and highly specific field, which will renovate the healthcare in near future which would enhance quality of life of patients. The scientific vision carried forward by nanotechnology is to open up opportunities for developing new diagnostic tools to provide the ability for people to test themselves and their health to be constantly monitored. Additionally, medical implants could take advantage of the improved knowledge on how materials like plastics and metals interact with the human body, assisting doctors to replace worn out body parts with artificial ones.

Ambitious scientific progress in nanotechnology, medical diagnosis will move to a phase in which straightforward disease diagnosis; independent of physician visits and large centralized laboratories. Indeed, further novel variations and innovation in nanotechnology will continue to appear and produce new opportunities for medical diagnosis. The ability of nanobiosensor to detect physiologically relevant biomolecules viz. glucose, cholesterol etc. and pathogens in the clinical samples with high sensitivity and specificity offers a benefits for early diagnosis and treatment of diseases.

\section{References}

1. The American Heritage $\circledast$ Dictionary of the English Language, 4th edition Copyright (C) 2010 by Houghton Mifflin Harcourt Publishing Company.

2. Azzazy HM, Mansour MM, Kazmierczak SC (2006) Nanodiagnostics: a new frontier for clinical laboratory medicine. Clin Chem 52: 1238-1246.

3. Blaufox MD (2002) An ear to the chest: An illustrated history of the evolution of the stethoscope. J R Soc Med. Dec 95: 626-627. 
Citation: Satvekar RK, Tiwale BM, Pawar SH (2014) Emerging Trends in Medical Diagnosis: A Thrust on Nanotechnology. Med chem 4: 407-416. doi:10.4172/2161-0444.1000172

4. Rontgen WC (1896) On a new kind of rays. Science 3: 227-231.

5. Challa S S R Kumar (2007) Nanomaterials for Medical Diagnosis and Therapy.

6. Gaster RS, Hall DA, Wang SX (2011) nanoLAB: an ultraportable, handheld diagnostic laboratory for global health. Lab Chip 11: 950-956.

7. Jain KK (2009) Chapter 2 Molecular Diagnostics as Basis of Personalized Medicine, Textbook of Personalized Medicine.

8. Thesis: Nanostructured Electrochemical Biosensors:Towards Point of Care Diagnostics, Brian Lam , Department of Chemistry, University of Toronto.

9. Richard S Gaster, Drew A Hall, Carsten H Nielsen, Sebastian J Osterfeld, Heng $\mathrm{Yu}$, et al. (2009) Matrix-insensitive protein assays push the limits of biosensors in medicine. Nature Medicine 15: 1327-1332.

10. Mascini M, Tombelli S (2008) Biosensors for biomarkers in medical diagnostics. Biomarkers 13: 637-657.

11. Rosen JE, Yoffe S, Meerasa A, Verma M, Gu FX (2011) Nanotechnology and Diagnostic Imaging: New Advances in Contrast Agent Technology. J Nanomedic Nanotechnol 2: 115.

12. Wickline SA, Neubauer AM, Winter P, Caruthers S, Lanza G (2006) Applications of nanotechnology to atherosclerosis, thrombosis, and vascular biology. Arterioscler Thromb Vasc Biol 26: 435-441.

13. Akhtari M, Bragin A, Cohen M, Moats R, Brenker F, et al. (2008) Functionalized magnetonanoparticles for MRI diagnosis and localization in epilepsy. Epilepsia 49: 1419-1430.

14. 14. Turvey SE, Swart E, Denis MC, Mahmood U, Benoist C,et al. (2005) Noninvasive imaging of pancreatic inflammation and its reversal in type 1 diabetes. J Clin Invest 115: 2454-2461

15. Alzaidi A, Linfeng Zhang, BajwaH (2012) Smart textiles based wireless ECG system ,Syst ems, Applications and Technology Conference (LISAT), IEEE Long Island 1-5.

16. Norio Taniguchi, On the basic concept of 'Nanotechnology', Proc. Intl. Prod. Eng. Tokyo, part II, Japan society of precision Engineering, 1974, pp 18-23

17. Richard Feynman (1960) Caltech Engineering and Science 23: 22-36.

18. Shi J, Votruba AR, Farokhzad OC, Langer R (2010) Nanotechnology in drug delivery and tissue engineering: from discovery to applications. Nano Lett 10: 3223-3230.

19. Kim BY, Rutka JT, Chan WC (2010) Nanomedicine. N Engl J Med 363: 24342443.

20. Tallury $P$, Malhotra A, Byrne LM, Santra $S$ (2010) Nanobioimaging and sensing of infectious diseases. Adv Drug Deliv Rev 62: 424-437.

21. Shinjini S, Mritunjai S, Gambhir IS (2008) Nanotechnology for Alzheimer's Disease Detection. Digest Journal of Nanomaterials and Biostructures 3: 7579.

22. Fakruddin M, Hossain Z, Afroz H (2012) Prospects and applications of nanobiotechnology: a medical perspective. J Nanobiotechnology 10: 31.

23. Chapter 6 Nanomaterials, Springer.

24. Pawar SH, Ghosh SJ, Karande VA, Patil RN (2011) Recent Advances in Microbial Synthesis of Nanoparticles and Their Applications. Book chapter In Nova Science Publishers, Inc.

25. Shao H, Yoon TJ, Liong M, Weissleder R, Lee H (2010) Magnetic nanoparticles for biomedical NMR-based diagnostics. Beilstein J Nanotechnol 1: 142-154.

26. Koh I, Josephson L (2009) Magnetic nanoparticle sensors. Sensors (Basel) 9: 8130-8145.

27. Hofmann-Amtenbrink M, Hofmann H, Montet X (2010) Superparamagnetic nanoparticles - a tool for early diagnostics. Swiss Med Wkly 140: w13081.

28. Azzazy HM, Mansour MM, Kazmierczak SC (2007) From diagnostics to therapy: prospects of quantum dots. Clin Biochem 40: 917-927.

29. Smith AM, Dave S, Nie S, True L, Gao X (2006) Multicolor quantum dots for molecular diagnostics of cancer. Expert Rev Mol Diagn 6: 231-244.

30. Xing Y, Rao J (2008) Quantum dot bioconjugates for in vitro diagnostics \& in vivo imaging. Cancer Biomark 4: 307-319.

31. Zhang Y, Bai Y, Yan B (2010) Functionalized carbon nanotubes for potential medicinal applications. Drug Discov Today 15: 428-435.

32. Leyden MR, Messinger RJ, Schuman C, Sharf T, Remcho VT, et al. (2012)
Increasing the detection speed of an all-electronic real-time biosensor. Lab Chip 12: 954-959.

33. Vashist SK, Zheng D, Al-Rubeaan K, Luong JH, Sheu FS (2011) Advances in carbon nanotube based electrochemical sensors for bioanalytical applications. Biotechnol Adv 169-188.

34. Dresselhaus MS, Araujo PT (2010) Perspectives on the 2010 Nobel Prize in physics for graphene. ACS Nano 4: 6297-6302.

35. Rajashekhar Kanchanapally, Zhen Fan, Anant Kumar Singh, Sudarson Sekhar Sinhaa et al. (2014) Multifunctional hybrid graphene oxide for labelfree detection of malignant melanoma from infected blood. J Mater Chem B 2: 1934-1937.

36. Sperling RA, Rivera Gil P, Zhang F, Zanella M, Parak WJ (2008) Biological applications of gold nanoparticles. Chem Soc Rev 37: 1896-1908.

37. Shanmukh S, Jones L, Zhao YP, Driskell JD, Tripp RA, et al. (2008) Identification and classification of respiratory syncytial virus (RSV) strains by surface-enhanced Raman spectroscopy and multivariate statistical techniques, Anal Bioanal Chem 390: 1551-1555.

38. Satvekar RK, Phadatare MR, Karande VA, Patil RN, Tiwale BM, et al. (2012) Influence of Silane Content on the Optical Properties of Sol Gel Derived Spin Coated Silica Thin Films. International Journal of Basic and Applied Sciences 1: 468-476.

39. Kim D, Fu C, Ling XB, Hu Z, Tao G, et al. (2013) Pilot Application of Magnetic Nanoparticle-Based Biosensor for Necrotizing Enterocolitis. J Proteomics Bioinform S5: 002.

40. Chung HJ, Castro CM, Im H, Lee H, Weissleder R (2013) A magneto-DNA nanoparticle system for rapid detection and phenotyping of bacteria. Nat Nanotechnol 8: 369-375.

41. Naveedul Hassan Alvi, Victor J. Gómez, Paul E.D. Soto Rodriguez, Praveen Kumar, SaimaZaman, et al. (2013) An InN/InGaN Quantum Dot Electrochemical Biosensor for Clinical Diagnosis,Sensors 13: 13917-13927.

42. MateiRaicopol, AlinaPruna, Celina Damian, Luisa Pilan (2013) Functionalized single-walled carbon nanotubes/polypyrrole composites for amperometric glucose biosensors. Nanoscale Research Letters 8: 316.

43. Anu Singh, Gaurav Sinsinbar, Meenakshi Choudhary, Veeresh Kumar, Renu Pasricha, et al. (2013) Graphene oxide-chitosan nanocomposite based electrochemical DNA biosensor for detection of typhoid. Sensors and Actuators B: Chemical 185: 675-684.

44. Zhu L, Xu L, Tan L, Tan H, Yang S, et al. (2013) Direct electrochemistry of cholesterol oxidase immobilized on gold nanoparticles-decorated multiwalled carbon nanotubes and cholesterol sensing. Talanta 106: 192-199.

45. Khadijeh Eskandari, Hedayatollah Ghourchian (2013) Performance of gold- and silver-coated magnetic nanoparticles as carriers for horseradish peroxidase, Journal of the Iranian Chemical Society 10: 1113-1121.

46. Padmaja Parameswaran Nampi, Cheranellor Chandrasekharan Kartha, Gin Jose, Anil Kumar PR, Thapasimuthu Anilkumar, et al. (2013) Sol-gel nanoporous silica as substrate for immobilization of conjugated biomolecules for application as fluorescence resonance energy transfer (FRET) based biosensor. Sensors and Actuators B: Chemical 185: 252-257.

47. Jain KK (2000) Applications of biochip and microarray systems in pharmacogenomics. Pharmacogenomics 1: 289-307.

48. Zhang YQ, Wang YF, Jiang XD (2008) The application of nanoparticles in biochips. Recent Pat Biotechnol 2: 55-59.

49. 49. Jing Feng,Vince S. Siu,Alec Roelke, Vihang Mehta, Steve Y. Rhieu, et al. (2012) Nanoscale Plasmonic Interferometers for Multispectral, HighThroughput Biochemical Sensing. Nano Lett 12: 602-609.

50. Zhu H, Snyder M (2003) Protein chip technology. Curr Opin Chem Biol 7: 5563.

51. Haab BB (2005) Antibody arrays in cancer research. Mol Cell Proteomics 4: 377-383.

52. Griffith R Freeman, Paul A. Raju, Scott M. Norton, lan D. Walton, Patrick C. Smith, et al. (2005) Use of Nanobarcodes Particles in Bioassays, NanoBiotechnology Protocols, Methods in Molecular Biolog 303: 73-83.

53. Zhou H, Roy S, Schulman H, Natan MJ (2001) Solution and chip arrays in protein profiling. Trends Biotechnol 19: S34-39.

54. David Andrew Davis, William Klein, Lei Chang (2006) Nanotechnology-Based Approaches to Alzheimer's Clinical Diagnostics, Nanoscape. 
Citation: Satvekar RK, Tiwale BM, Pawar SH (2014) Emerging Trends in Medical Diagnosis: A Thrust on Nanotechnology. Med chem 4: 407-416. doi:10.4172/2161-0444.1000172

55. Jakeway SC, de Mello AJ, Russell EL (2001) Miniaturised total analysis systems for biological analysis (Review), Fresenius Journal of analytical chemistry 366 : 525-566.

56. Attila Roka and H. Schoenfeld (2011) Remote Monitoring of Cardiac Implantable Electronic Devices, The Journal of Innovations in Cardiac Rhythm Management 2: 381-393.

57. HuangxianJu, Xueji Zhang, Joseph Wang, NanoBiosensing Principles, Development and Application, Biological and Medical Physics, Biomedical Engineering.

58. Turner AP (2013) Biosensors: sense and sensibility. Chem Soc Rev 42: $3184-$ 3196.

59. Thévenot DR1, Toth K, Durst RA, Wilson GS (2001) Technical report: Electrochemical biosensors: recommended definitions and classification. Biosens Bioelectron 16: 121-131.

60. CLARK LC Jr, LYONS C (1962) Electrode systems for continuous monitoring in cardiovascular surgery. Ann N Y Acad Sci 102: 29-45.

61. Connolly P (1995) Clinical diagnostics opportunities for biosensors and bioelectronics. Biosens Bioelectron 10: 1-6.

62. Habermuller K, Mosbach M, Schuhmann W (2000) Electron-transfer mechanisms in amperometric biosensors. Fresenius J Anal Chem 366: 560 568.

63. Bakker E (2004) Electrochemical sensors. Anal Chem 76: 3285-3298.

64. Zarini Muhammad-Tahir, and Evangelyn C. Alocilja (2003) A conductometric biosensor for biosecurity. Biosensors and Bioelectronics 18: 813-819.

65. Dey D, Goswami T (2011) Optical Biosensors: A Revolution Towards Quantum Nanoscale Electronics Device Fabrication, J Biomed Biotechnol 348218.

66. Kim N, Park IS, Kim DK (2004) Characteristics of label-free piezoelectric immunosensor detecting Pseudomonas aeruginosa, Sensors \& Actuators B, Chemical 100: 432-438.
67. Cooper MA (2003) Label-free screening of bio-molecular interactions. Anal Bioanal Chem 377: 834-842.

68. Xie B, Ramanathan K, Danielsson B (1999) Principles of Enzyme Thermistor Systems: Applications to Biomedical and Other Measurements, Adv Biochem Eng Biotechnol 64:1-33.

69. Wang J (2002) Electrochemical nucleic acid biosensors. AnalyticaChimicaActa 469: 63-71.

70. Stefan RI, van Staden JF, Aboul-Enein HY (2000) Immunosensors in clinical analysis. Fresenius J Anal Chem 366: 659-668.

71. Sassolas A, Blum LJ, Leca-Bouvier BD (2012) Immobilization strategies to develop enzymatic biosensors. Biotechnol Adv 30: 489-511.

72. Belkin S, Gu MB (2010) Whole Cell Sensing Systems I: Reporter Cells and Devices. Springer, 40 Hiedelberg.

73. Rajshri KS, Rohiwal SS, Raut AV, Karande VA, Tiwale BM, et al. (2014) A silica-dextran nanocomposite as a novel matrix for immobilization of horseradish peroxidase, and its application to sensing hydrogen peroxide. Microchimicaacta 181: 71-77

74. Scognamiglio V (2013) Nanotechnology in glucose monitoring: advances and challenges in the last 10 years. Biosens Bioelectron 47: 12-25.

75. Arya SK, Datta M, Malhotra BD (2008) Recent advances in cholesterol biosensor. Biosens Bioelectron 23: 1083-1100.

76. DSouza SF, Jitendra K, Sandeep K, Kubal BS (2013) Immobilization of the urease on eggshell membrane and its application in biosensor. Materials Science and Engineering C 33: 850-854.

77. Gonzalez-Macia L, Morrin A, Smyth MR, Killard AJ (2010) Advanced printing and deposition methodologies for the fabrication of biosensors and biodevices. Analyst 135: 845-867. 